\title{
MULTIPLE AUTOIMMUNE SYNDROMES ASSOCIATED WITH THYMIC MALIGNANCY
}

Carlos Augusto Rodrigues Padilha ${ }^{2, \star}$, Danielly Dantas Pimentel², Gustavo Roberto Lourenço², Juliana de Jesus Boscolo², lane Tamara Dondé ${ }^{2}$, Maria Juliana da Silva Almeida², Patrícia Milani de Moraes², Renata Vaz de Oliveira², Taísa Morete da Silva², Carla Lionela Trigo Romero ${ }^{1}$

1. Faculdade de Medicina de Ribeirão Preto, Ribeirão Preto (SP), Brazil. 2. Faculdade de Medicina de São José do Rio Preto, São José do Rio Preto (SP), Brazil.

${ }^{\star}$ Corresponding author: rpadilha.carlos@gmail.com

\section{BACKGROUND}

Thymomas are often associated with paraneoplastic disorders. As the thymus has a central role in the immune system, autoimmune syndromes related to thymic malignancy are expected. About $30 \%$ of thymoma patients will develop autoimmune conditions, despite thymectomy, and $50 \%$ of patients are diagnosed with more than one autoimmune disorder at the same time.

\section{CASE REPORT}

A 32-year-old woman was admitted to our service with a 5-month history of fatigue, jaundice, nausea, diarrhea, and right upper quadrant abdominal pain. Laboratory tests found elevated liver function tests (elevated transaminase and canalicular enzymes levels and hyperbilirubinemia). Abdominal ultrasound showed enlarged liver (hepatomegaly) with heterogeneous echogenicity and intrahepatic duct dilatation, and hepatic biopsy revealed highly active chronic hepatitis, with porto-portal and porto-central septa, compatible with autoimmune hepatitis. Immunosuppression treatment with $40 \mathrm{mg}$ prednisone and 50 mg azathioprine was initiated, with subsequent patient improvement and discharge. Due to a complaint of dryness of the eyes, outpatient rheumatology follow-up was initiated. A positive Schirmer's test, presence of anti-SSA antibodies, hypergammaglobulinemia, and antinuclear antibodies (ANA) nuclear homogeneous pattern with a titer 1/1280 was compatible with Sjögren's syndrome diagnosis. During the

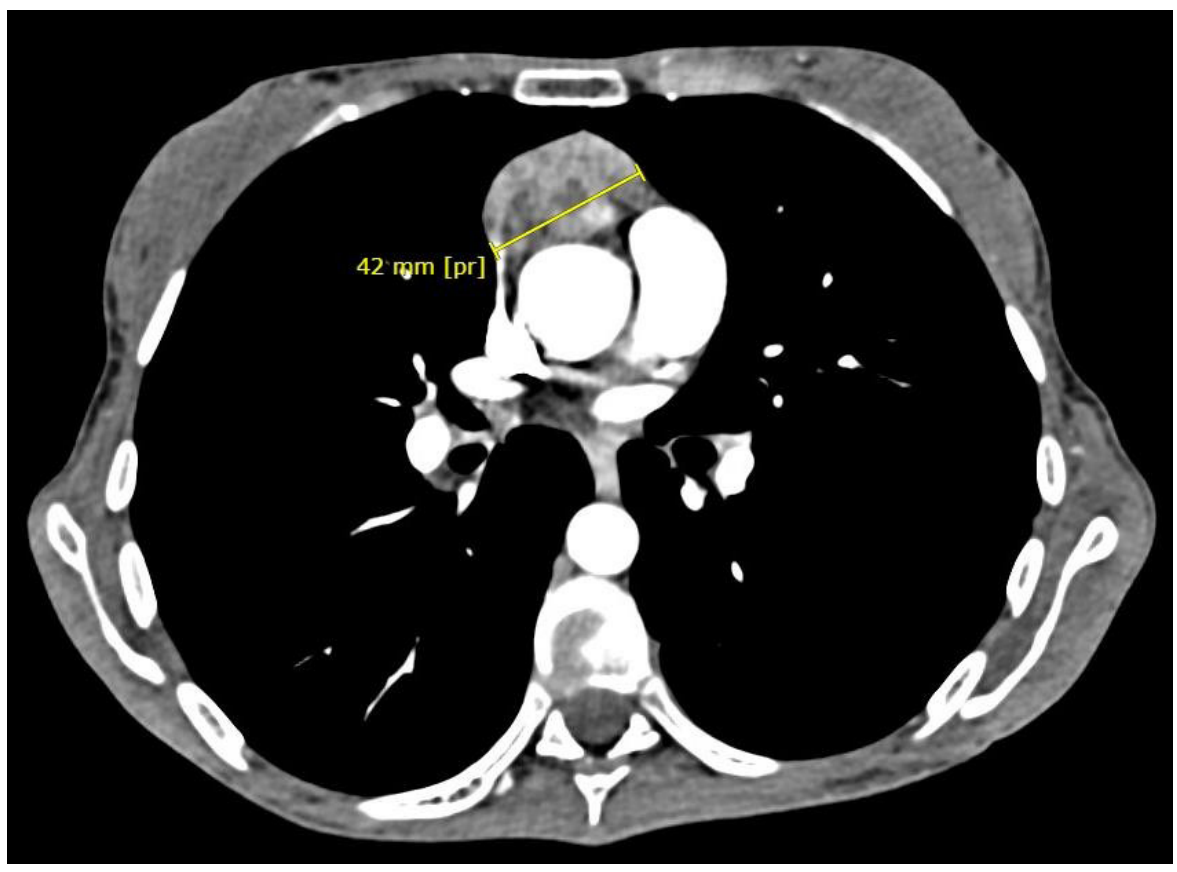

Figure 1. Computed tomography image of the mediastinal mass. The yellow line indicates the size of the mass in a transversal section. 
investigation, thyroid function was normal, but antithyroglobulin was positive $(1374 \mathrm{UI} / \mathrm{mL})$. Seven months after the first admission, the patient was readmitted due to a 35-day steatorrhea and 5-kg loss. Abdominal computed tomography (CT) showed colon, sigmoid, and rectum wall thickness, diffuse parietal enhancement, and mild diffuse distension of bowel's walls. An intestinal biopsy reported moderate chronic nonspecific colitis with mild neutrophilic activity and reactional follicular lymphoid hyperplasia. Two months later, a new abdominal CT scan showed signs of intussusception, and the patient was submitted to a right hemicolectomy. Thoracic CT scan showed a heterogeneous formation in the anterior mediastinum (Figure 1 ) measuring $4.2 \times 2.8 \mathrm{~cm}$ with necrotic areas and post-contrast enhancement. Mediastinal mass biopsy showed a lesion compatible with thymoma type B1. Thymectomy was performed in the same month. Currently, the patient is receiving adjuvant radiation therapy with hepatic improvement, without immunosuppression therapy.

\section{CONCLUSION}

Among thymoma's paraneoplastic autoimmune syndromes, multiple rheumatologic disorders are described (systemic lupus erythematosus, rheumatoid arthritis, Sjögren's syndrome, scleroderma). We have described a case of a patient with hepatic, gastrointestinal, thyroid autoimmune alterations, and Sjögren's syndrome diagnosis. Rheumatologists need to be aware of this diagnosis, especially in cases where more than one autoimmune disease is diagnosed at the same time, and the patient has an unexpected disease age onset.

\section{KEYWORDS}

Sjögren's syndrome, Thymoma, Autoimmunity. 